\title{
38 Research Square

\section{MicroRNA Expression in Circulating Leukocytes and Bioinformatic Analysis of Patients with Moyamoya Disease}

\section{Kaijiang Kang}

Beijing Tiantan Hospital, Capital Medical University

\section{Yuan Shen}

Beijing Tiantan Hospital, Capital Medical University

Qian Zhang

Beijing Tiantan Hospital, Capital Medical University

Jingjing Lu

Beijing Tiantan Hospital, Capital Medical University

Yi Ju

Beijing Tiantan Hospital, Capital Medical University

\section{Ruijun Ji}

Beijing Tiantan Hospital, Capital Medical University

$\mathrm{Na} \mathrm{Li}$

Beijing Tiantan Hospital, Capital Medical University

Jianwei Wu

Beijing Tiantan Hospital, Capital Medical University

\section{Bo Yang}

Beijing Tiantan Hospital, Capital Medical University

Jinxi Lin

China National Clinical Research Center for Neurological Diseases

\section{Xianhong Liang}

China National Clinical Research Center for Neurological Diseases

\section{Dong Zhang}

Beijing Tiantan Hospital, Capital Medical University

\section{Xingquan Zhao ( $\nabla$ zxq@vip.163.com )}

Beijing Tiantan Hospital, Capital Medical University

\section{Research Article}

Keywords: Stroke, Cerebrovascular disorders, Cerebral infarction, Hemorrhage, Genetics 
Posted Date: September 2nd, 2021

DOI: https://doi.org/10.21203/rs.3.rs-778159/v1

License: (c) (i) This work is licensed under a Creative Commons Attribution 4.0 International License. Read Full License 


\section{Abstract}

Background and purpose-MicroRNAs (miRNAs) in exosomes had been implicated differentially expressed in MMD patients, but the miRNAs expression in circulating leukocytes remains unclear. This study was investigated on the differential expression of miRNAs in peripheral leukocytes between MMD patients and healthy adults, and among patients with subtypes of MMD.

Methods- $A$ total of 30 patients with MMD and 10 healthy adults were enrolled in a stroke center from October 2017 to December 2018. The gene microarray was used to detect the differential expression profiles of miRNA in leukocytes between MMD patients and controls, and the differentially expressed miRNAs were verified by the method of real-time PCR. The Gene Ontology (GO) and Kyoto Encyclopedia of Genes and Genomes (KEGG) were used to explore the key signaling pathways and possible pathogenesis of MMD.

Results- The microarray results showed 12 differentially expressed miRNAs in leukocytes of MMD patients compared with controls (fold change $>2.0$ and $P<0.05$ ), of which 7 miRNAs were up-regulated (miRNA-142-5p, miRNA-29b-3p, miRNA-424-5p, MiRNA-582-5p, miRNA-6807-5p, miRNA-142-3p, miRNA340-5p), and 5 miRNAs were down-regulated (miRNA-144-3p, miRNA-144-5p, miRNA-451a, miRNA-486-5p, miRNA-363-3p). The real-time PCR confirmed 7 differentially expressed miRNAs $(P<0.05)$, of which 4 miRNAs (miRNA-29b-3p, miRNA-142-3p, miRNA-340-5p, miRNA-582-5p) were up-regulated, and 3 miRNAs (miRNA-363-3p, miRNA-451a and miRNA-486-5p) were down-regulated. Both GO and KEGG analysis suggested that the Wnt signaling pathway may be involved in the pathogenesis of MMD. In addition, miRNAs were also differentially expressed among patients with subtypes of MMD.

Conclusion-This study indicated that miRNAs are differentially expressed in peripheral leukocytes between MMD patients and healthy adults, and among patients with subtypes of MMD. The Wnt signaling pathway is probably involved in the pathogenesis of MMD.

\section{Introduction}

Moyamoya disease (MMD) is a relatively rare cerebrovascular disease associated with recurrent stroke, and the prevalence of MMD have increased around the world, especially in Japan, Korea, and China[1-6]. Currently, the etiology and pathogenesis of MMD are still unclear, so there is a lack of reliable molecular biological markers and effective drugs[7-9]. The incidence of MMD has been demonstrated as racial propensity and familial clustering, and some genes have been indicated to be abnormally expressed, which suggests that the MMD may be closely associated with gene expressions[8-12]. MMD can be manifested as ischemic, hemorrhagic, or asymptomatic, and hemorrhagic stroke is one of the main factors leading to acute death and severe disability in patients with $\operatorname{MMD}[13,14]$. However, the mechanisms and predictors of hemorrhagic stroke associated with MMD are still limited to the vascular or hemodynamic characteristics, while the underlying pathophysiological mechanisms and biological processes are still unclear[15-18]. 
MicroRNA (miRNA) is a kind of endogenous small RNA with a length of about 18-24 nucleotides, which plays an important regulatory role in the normal metabolism of cells and the development of diseases by inhibiting the translation of messenger RNA (mRNA) into proteins or promoting the degradation of mRNA[19, 20]. Although miRNA has been investigated for several years, its relationship with MMD remains to be elucidated. It has been indicated that miRNAs were abnormally expressed in peripheral blood and cerebrospinal fluid of patients with MMD[21-24]. However, previous studies had mostly focused on the differences of miRNAs in exosomes, which cannot fully reflect the actual expression of intracellular miRNAs. Also, studies have shown that some mononuclear cells in peripheral blood can differentiate into vascular endothelial progenitor cells and vascular smooth muscle progenitor cells, and the pathophysiological process of MMD is often accompanied by inflammatory changes involving a variety of cytokines, suggesting that MMD may be closely associated with peripheral leukocytes $[25,26]$.

In this study, we investigated the differential expression of miRNA in peripheral leukocytes between MMD patients and healthy adults, and among patients with subtypes (hemorrhagic, ischemic, and asymptomatic) of MMD, to explore the pathogenesis of MMD, and provide the basis for clinical diagnosis, outcome prediction and therapeutic strategy of MMD.

\section{Materials And Methods Study population}

The study was performed according to the guidelines from the Helsinki Declaration, and was approved by the Research Ethics Committee of the hospital. Written informed consent was obtained from all the subjects or their legally authorized representatives. A total of 30 patients, who were diagnosed with MMD in our stroke center from October 2017 to December 2018, were consecutively included in this study. The inclusion criteria included: 1) the age was 18-65 years; 2) diagnosed as MMD according to the 2012 Japanese Moyamoya disease diagnosis and treatment guidelines[27]; 3) the patient's informed consent to the study protocol. The exclusion criteria included: 1) moyamoya syndrome indicated by clinical manifestations, high-resolution MRI, or laboratory examinations; 2 ) with history of stroke in the past three months; 3 ) patients who had received revascularization surgery; 4) patients with immunological diseases, tumors, or pregnancy. In addition, 10 healthy adults with matching gender and age were included as normal controls.

\section{Sample collection and RNA extraction}

Peripheral venous blood samples (about $6 \mathrm{ml}$ ) were taken from the antecubital vein into an anticoagulant drying tube under fasting conditions. The whole blood was centrifuged at $2600 \mathrm{rpm}$ for $10 \mathrm{~min}$ at room temperature within an hour. The white blood cells were separated from the middle layer, and the mixed red blood cells were removed with red blood cell lysis buffer. A kind of RNA stabilizer (RNAlater, Thermo Fisher Scientific, Baltics UAB, Vilnius, Lithuania) was added to the cryotube containing white blood cells 
and was stored at $4{ }^{\circ} \mathrm{C}$ for 24 hours, then stored in $-80^{\circ} \mathrm{C}$ refrigerator for standby. Trizol Reagent (Invitrogen, Carlsbad, CA, USA) was used for leukocyte denaturation. The miRNeasy Mini Kit (Qiagen p/n 217004) was used for RNA collection and purification according to the manufacturer's protocol. The quality evaluation of the total RNA was performed using the Agilent 2100 Bioanalyzer (Agilent, Santa Clara, CA, USA).

\section{miRNA microarray}

The Agilent Human (8*60K) V21.0 miRNA microarrays (Agilent, Santa Clara, CA, USA) were performed using a Gene Expression Hybridization Kit (Agilent's miRNA Complete Labeling and Hyb Kit-p/n51900456) according to the manufacturer's instructions. Slides were washed in staining dishes with a Gene Expression Wash Buffer Kit (Agilent, Santa Clara, CA, USA) and scanned by an Agilent Microarray Scanner with default settings according to the manufacturer's instructions. The data was extracted using an Agilent Feature Extraction (AFE) software (version 10.7.1.1), and the raw data were normalized by Quantile algorithm using Gene Spring Software 12.6 (Agilent Technologies). Differentially expressed miRNAs were identified through fold-change $(>2)$ and $P$ value $(<0.05)$ filtering using R software (version 3.2.3) with the samr package.

\section{Real-time PCR}

Several differentially expressed miRNAs were quantified by using real-time polymerase chain reaction (RTPCR) based on the Taqman method. Total RNAs were reversely transcribed using the Taqman MicroRNA Reverse Transcription Kit (ABI, 4366597) according to the manufacturer's instructions. RT-PCR was performed using QuantStudio 5 Real-Time PCR System (ABI, USA) and QuantStudio ${ }^{\text {TM }}$ Design \& Analysis Software with the Taqman Universal PCR Master Mix (ABI, 4440049). The program was as follows: $95^{\circ} \mathrm{C}$, $10 \mathrm{~min}$; $\left(95^{\circ} \mathrm{C}, 15 \mathrm{~s} ; 60^{\circ} \mathrm{C}, 1 \mathrm{~min}\right) 40$ cycles. The expression level of each miRNA was calculated as fold change adopting the $2^{-\Delta \Delta C t}$ method. We conducted three replicate experiments to test the reproducibility. The homogenous miRNA-16 was used as an internal reference for the analysis.

\section{Bioinformatics analysis}

In this study, we used TargetScan (http://www.targetscan.org/, TargetScanHuman 7.2) to identify the targets of differentially expressed miRNAs. We defined the predicted target genes by no less than 10 differentially expressed miRNAs as potential functional targets in MMD. To determine the biological relationship between the potential miRNA target genes, the Gene Ontology (GO) and Kyoto Encyclopedia of Genes and Genomes (KEGG) were performed using DAVID Bioinformatics Resources (http://david.abcc.ncifcrf.gov/, DAVID Bioinformatics Resources 6.8). The significance threshold was set to 0.05 in our enrichment analysis. 


\section{Statistical analysis}

The statistical analysis was performed using SPSS (version 22.0, IBM-SPSS, Chicago, IL, USA) and R software (version 3.2.3). Genes with a two-sided $P$ value of $<0.05$ and fold change $>2.0$ were regarded as statistically significant genes.

\section{Results}

\section{Demographic information and clinical characteristics}

The study recruited 30 patients with MMD (including 10 hemorrhagic MMD patients, 12 ischemic MMD patients, and 8 asymptomatic MMD patients), and 10 healthy adults with matched demographic characteristics. The detailed characteristics of the included subjects were shown in Table 1.

\section{miRNA microarray}

In analysis of $30 \mathrm{MMD}$ patients compared with 10 controls, 9 miRNAs were found to be differentially expressed, with 5 up-regulated (miRNA-142-5p, miRNA-29b-3p, miRNA-424-5p, miRNA-582-5p, miRNA6807-5p) and 4 down-regulated (miRNA-144-3p, miRNA-144-5p, miRNA-451a, miRNA-486-5p) (Table 2, Figure 1). In addition, miRNA expression was significantly different in 6 samples (4 in the MMD group and 2 in the control group). After the elimination of these 6 samples, miRNA-142-3p, miRNA-340-5p, miRNA-363-3p were also differentially expressed in MMD patients (miRNA-142-3p and miRNA-340-5p upregulated, while miRNA-363-3p down-regulated).

In analysis of 10 hemorrhagic MMD patients compared with 10 controls, 17 miRNAs were found to be differentially expressed, with 13 up-regulated (miRNA-101-3p, miRNA-142-3p, miRNA-142-5p, miRNA148a-3p, miRNA-29b-3p, miRNA-340-5p, miRNA-374a-5p, miRNA-424-5p, miRNA-450a-5p, miRNA-542-3p, miRNA-582-5p, miRNA-590-5p, miRNA-6807-5p) and 4 down-regulated (miRNA-144-3p, miRNA-144-5p, miRNA-451a, miRNA-486-5p). In analysis of 8 asymptomatic MMD patients compared with 10 controls, 7 miRNAs were found to be differentially expressed, with 3 up-regulated (miRNA-142-3p, miRNA-29b-3p, miRNA-6515-5p) and 4 down-regulated (miRNA-144-3p, miRNA-144-5p, miRNA-451a, miRNA-486-5p). In analysis of 12 ischemic MMD patients compared with 10 controls, 3 miRNAs were found to be differentially expressed (miRNA-29b-3p and miRNA-424-5p up-regulated, while miRNA-486-5p downregulated) (Figure 2).

In addition, miRNA-486-5p was found to be differentially down-regulated in hemorrhagic MMD patients, compared with ischemic MMD patients. Also, miRNA-6515-5p was found to be differentially up-regulated in asymptomatic MMD patients, compared with symptomatic (hemorrhagic and ischemic) MMD patients. 


\section{Real-time PCR}

The RT-PCR verification of 10 differentially expressed miRNAs was performed between 30 MMD patients and 10 controls, including miRNA-142-5p, miRNA-29b-3p, miRNA-424-5p, miRNA-582-5p, miRNA-144-3p, miRNA-451a, miRNA-486-5p, miRNA-142-3p, miRNA-340-5p, miRNA-363-3p. The probe was synthesized by Thermo Fisher Scientific, and the target sequence of the probe is shown in Table 3. Three independent experiments had strong reproducibility, and all of the 10 genes were identified successfully. However, two normal control samples had higher overall CT values than other samples, but miRNA-16 (internal reference) was relatively lower, which led to higher overall expression and affected the overall analysis results, so the two samples were removed during the final analysis.

Compared with 10 healthy adults, 7 miRNAs had differential expression in 30 MMD patients $(P<0.05)$, of which miRNA-29b-3p ( $P<0.001)$, miRNA-142-3p ( $P=0.013)$, miRNA-340-5p ( $P=0.002)$, miRNA-582-5p $(P=0.005)$ was up-regulated, while miRNA-363-3p $(P=0.007)$, miRNA-451a $(P<0.001)$, miRNA-486-5p $(P=0.001)$ was down-regulated (Figure 3$)$.

Compared with 10 healthy adults, 9 miRNAs had differential expression in 10 hemorrhagic MMD patients $(P<0.05)$, of which miRNA-29b-3p ( $P=0.002)$, miRNA-142-3p ( $P=0.004)$, miRNA-142-5p ( $P=0.047)$, miRNA340-5p $(P<0.001)$, miRNA-582-5p $(P=0.003)$ were up-regulated, while miRNA-451a $(P=0.001)$, miRNA-486$5 p(P=0.035)$, miRNA-144-3p ( $P=0.009)$, miRNA-363-3p $(P=0.002)$ were down-regulated. Compared with 10 healthy adults, 6 miRNAs had differential expression in 8 asymptomatic MMD patients $(P<0.05)$, of which miRNA-29b-3p ( $P=0.004)$, miRNA-142-3p ( $P=0.001)$, miRNA-340-5p ( $P=0.009)$, miRNA-582-5p $(P=0.005)$ were up-regulated, while miRNA-363-3p $(P=0.015)$, miRNA-451a $(P=0.009)$ were downregulated. Compared with 10 healthy adults, 4 miRNAs had differential expression in 12 ischemic MMD patients $(P<0.05)$, of which miRNA-29b-3p $(P=0.006)$, miRNA-142-5p $(P=0.035)$, miRNA-340-5p $(P=0.030)$ were up-regulated, while miRNA-451a $(P=0.030)$ was down-regulated.

In addition, the miRNA-142-3p was up-regulated in hemorrhagic MMD patients and miRNA-340-5p was up-regulated in hemorrhagic and the asymptomatic MMD patients compared with ischemic MMD patients $(P<0.05)$. The miRNA-486-5p was down-regulated in hemorrhagic and asymptomatic MMD patients, but there was no statistically significant difference $(P>0.05)$. There was no significant differential expression between the hemorrhagic and the asymptomatic MMD patients.

\section{Target gene prediction and bioinformatics analysis}

We used the TargetScan target gene prediction program to predict the potential target genes of differentially expressed miRNAs by microarray and RT-PCR. According to the 7 differentially expressed miRNAs in peripheral leukocytes of MMD patients, a total of 3728 target genes were predicted. Of the 3728 target genes, the biological processes and signal transduction pathways that may be involved in the enrichment analysis are carried out through GO and KEGG. The associated biological processes and signal pathways were screened (according to the criteria: $P$ value $\leq 0.05$, number of target genes $\geq 10$ ) 
and sequenced according to the enrichment degree, and the top 30 biological processes and signal pathways are shown in Figure 4. Both the GO and KEGG analysis suggested that the Wnt signaling pathway may be involved in the pathogenesis of MMD (Figure 4, Figure 5).

Table 1

Demographic and clinical characteristics of the subjects 


\begin{tabular}{|c|c|c|c|c|c|}
\hline \multirow[t]{2}{*}{ ID } & \multirow[t]{2}{*}{ Subtypes } & \multirow[t]{2}{*}{ Gender } & \multirow[t]{2}{*}{ Age (years) } & \multicolumn{2}{|c|}{ Suzuki stage } \\
\hline & & & & Left & Right \\
\hline HM_01 & Hemorrhagic MMD & Female & 32 & 4 & 4 \\
\hline HM_02 & Hemorrhagic MMD & Female & 43 & 2 & 3 \\
\hline HM_03 & Hemorrhagic MMD & Female & 24 & 3 & 3 \\
\hline HM_04 & Hemorrhagic MMD & Female & 49 & 6 & 6 \\
\hline HM_05 & Hemorrhagic MMD & Male & 49 & 6 & 3 \\
\hline HM_06 & Hemorrhagic MMD & Male & 42 & 5 & 5 \\
\hline HM_07 & Hemorrhagic MMD & Female & 50 & 3 & 3 \\
\hline HM_08 & Hemorrhagic MMD & Female & 34 & 3 & 3 \\
\hline HM_09 & Hemorrhagic MMD & Male & 50 & 0 & 3 \\
\hline HM_10 & Hemorrhagic MMD & Female & 42 & 4 & 4 \\
\hline IM_01 & Ischemic MMD & Male & 34 & 3 & 3 \\
\hline IM_02 & Ischemic MMD & Female & 36 & 3 & 1 \\
\hline IM_03 & Ischemic MMD & Female & 46 & 3 & 3 \\
\hline IM_04 & Ischemic MMD & Male & 35 & 3 & 3 \\
\hline IM_05 & Ischemic MMD & Female & 30 & 4 & 3 \\
\hline IM_06 & Ischemic MMD & female & 46 & 3 & 3 \\
\hline IM_07 & Ischemic MMD & Male & 47 & 4 & 3 \\
\hline IM_08 & Ischemic MMD & Female & 21 & 2 & 3 \\
\hline IM_09 & Ischemic MMD & Female & 29 & 2 & 2 \\
\hline IM_10 & Ischemic MMD & Male & 39 & 5 & 4 \\
\hline IM_11 & Ischemic MMD & Male & 37 & 2 & 2 \\
\hline IM_12 & Ischemic MMD & Male & 39 & 2 & 2 \\
\hline AM_01 & Asymptomatic MMD & Male & 42 & 2 & 2 \\
\hline AM_02 & Asymptomatic MMD & Male & 34 & 5 & 3 \\
\hline AM_03 & Asymptomatic MMD & Female & 29 & 3 & 3 \\
\hline AM_04 & Asymptomatic MMD & Female & 38 & 3 & 3 \\
\hline AM_05 & Asymptomatic MMD & Female & 34 & 4 & 4 \\
\hline
\end{tabular}




\begin{tabular}{llllll} 
AM_06 & Asymptomatic MMD & Female & 48 & 2 & 2 \\
\hline AM_07 & Asymptomatic MMD & Male & 50 & 3 & 3 \\
\hline AM_08 & Asymptomatic MMD & Female & 33 & 6 & 5 \\
\hline C_01 & Control & Male & 42 & - & - \\
\hline C_02 & Control & Male & 35 & - & - \\
\hline C_03 & Control & Male & 35 & - & - \\
\hline C_04 & Control & Male & 34 & - & - \\
\hline C_05 & Control & Female & 34 & - & - \\
\hline C_06 & Control & Female & 32 & - & - \\
\hline C_07 & Control & Female & 33 & - & - \\
C_08 & Control & Female & 35 & - & - \\
C_09 & Control & Female & 31 & - & - \\
C_10 & Control & Female & 33 & - & -
\end{tabular}

HM: hemorrhagic moyamoya disease; IM: ischemic moyamoya disease; AM: asymptomatic moyamoya disease; C: controls

Table 2

Differentially expressed miRNAs in microarray analysis between $30 \mathrm{MMD}$ patients and 10 controls

\begin{tabular}{|llll|}
\hline Systematic Name & P values & Fold change (abs) & Regulation \\
\hline miRNA-142-5p & 0.000020 & 2.020448 & up \\
\hline miRNA-144-3p & 0.000958 & 2.043367 & down \\
\hline miRNA-144-5p & 0.002665 & 2.413351 & down \\
\hline miRNA-29b-3p & 0.000006 & 2.420699 & up \\
\hline miRNA-424-5p & 0.000043 & 2.309035 & up \\
\hline miRNA-451a & 0.000068 & 2.478956 & down \\
\hline miRNA-486-5p & 0.000165 & 5.521476 & down \\
\hline miRNA-582-5p & 0.001293 & 2.228220 & up \\
\hline miRNA-6807-5p & 0.000010 & 2.227829 & up \\
\hline
\end{tabular}


Table 3

Target sequence of the probe in real-time PCR

\begin{tabular}{|ll|}
\hline miRNA & Target sequence of the probe \\
\hline miRNA-16 & UAGCAGCACGUAAAUAUUGGCG \\
\hline miRNA-29b & UAGCACCAUUUGAAAUCAGUGUU \\
\hline miRNA-142-3p & UGUAGUGUUUCCUACUUUAUGGA \\
\hline miRNA-142-5p & CAUAAAGUAGAAAGCACUACU \\
\hline miRNA-144 & UACAGUAUAGAUGAUGUACU \\
\hline miRNA-340 & UUAUAAAGCAAUGAGACUGAUU \\
\hline miRNA-363 & AAUUGCACGGUAUCCAUCUGUA \\
\hline miRNA-424 & CAGCAGCAAUUCAUGUUUUGAA \\
\hline miRNA-451 & AAACCGUUACCAUUACUGAGUU \\
\hline miRNA-486 & UCCUGUACUGAGCUGCCCCGAG \\
\hline miRNA-582-5p & UUACAGUUGUUCAACCAGUUACU \\
\hline
\end{tabular}

\section{Discussions}

The results of this study suggested that miRNA-29b-3p, miRNA-142-3p, miRNA-340-5p, and miRNA-582$5 p$ were significantly up-regulated, while miRNA-363-3p, miRNA-451a, miRNA-486-5p were significantly down-regulated in peripheral leukocytes of patients with MMD compared with healthy controls. In addition, miRNAs were also differentially expressed among patients with subtypes of MMD.

Previous studies had suggested that miRNA-106b, miRNA-130a, miRNA-126, miRNA-125-3p, miRNA-196a, let-7c were abnormally expressed in the peripheral serum of MMD patients[21, 23, 24]. Also, the miRNA3679-5p, miRNA-6165, miRNA-6760-5p, and miRNA-574-5p had been indicated to be differentially expressed in the cerebrospinal fluid of MMD patients[22]. The miRNA-196a was suggested to regulate cell proliferation and apoptosis by regulating the expression of ANXA1 gene in endothelial cells and vascular smooth muscle cells, which may be related to the onset of MMD[23]. It was indicated that the serum let7c in MMD patients was significantly increased, which can bind to the 3'non-coding region of RNF213 transcribed mRNA, thereby affecting the biological activity of RNF213[21]. The RNF213 mutation is currently considered to be a susceptible gene for MMD in Asian populations, which may be involved in the pathogenesis of MMD[28]. In the present study, however, these miRNAs did not show abnormal expression in peripheral leukocytes of MMD patients compared with controls, which suggested that there may be significant differences in the expression of miRNA between peripheral leukocytes and serum of patients with MMD. The circulating serum miRNAs reflect only the level of extracellular miRNAs secreted 
by a variety of cells through exosomes, but cannot fully reflect the true expression of specific intracellular miRNAs, which impeded further investigations on the miRNA-related target genes and cell functions.

The pathological characteristics of MMD are mainly manifested as a gradual degeneration of the smooth muscle in the arterial media, and the abnormal proliferation of the smooth muscle in the arterial intima, leading to progressive stenosis and occlusion of the involved vessels, accompanied by the development of collaterals[7-9]. The present study indicated that several biological processes or signal transduction pathways may be involved in the pathophysiological process of MMD, including vascular smooth muscle cell proliferation and metastasis, apoptosis, neovascularization, immune and inflammatory reactions, and thrombosis.

Both the GO analysis and KEGG analysis suggested that the Wnt signaling pathway may be involved in the pathogenesis of MMD (Fig. 4, Fig. 5). The Wnt signaling pathway is a complex regulatory network consisting of the secreted Wnt protein family, the transmembrane receptor Frizzled family, CK1, Deshevelled, GSK3, APC, Axin, $\beta$-catenin, and TCF/Lef family[29]. When the extracellular ligand binds to the cell surface receptor, the intracellular segment of the surface receptor was activated, which transmits extracellular signals into the cells. Previous studies have shown that the Wnt signaling pathway is activated in some cardiovascular and cerebrovascular diseases, and plays an important role in the proliferation, migration, apoptosis, and differentiation of vascular smooth muscle cells, which is one of the mechanisms of MMD[30-33]. Therefore, the role of the Wnt signaling pathway in MMD is worthy of further investigation.

In addition, miRNAs were also differentially expressed among patients with subtypes of MMD. Patients with MMD need to undergo a chronic conversion of cerebral hemodynamics and feeding arteries, from the original internal carotid artery system to the external carotid artery system or vertebrobasilar artery system. Depending on the degree or weight of vascular occlusion and collateral vasodilation, MMD can be manifested as ischemic, asymptomatic, or hemorrhagic $[8,9]$. In this study, the microarray results demonstrated an increasing number of differentially expressed miRNAs from ischemic MMD to asymptomatic MMD to hemorrhagic MMD compared with controls. Also, the real-time PCR results suggested that the miRNA-142-3p was up-regulated in hemorrhagic MMD patients, and the miRNA-340-5p was up-regulated in hemorrhagic and asymptomatic MMD patients compared with ischemic MMD patients. This indicated that miRNA may be involved in the process of arterial occlusion and collateral development.

In this study, we found that the miRNAs are abnormally expressed in peripheral leukocytes of patients with MMD. After further verification of real-time PCR with larger sample size, and verification of target genes regulation and cell function regulation, these differentially expressed miRNAs are likely to become new molecular biological markers of MMD and associated stroke, and hold a promise to become new potential targets of therapeutic strategy.

Potential limitations of our studies should be mentioned. First, all of the patients in this study were enrolled from a single center, so potential selection bias may be inevitable. We, however, tried our best to 
reduce in-house selection bias by collecting patients consecutively. Second, the case-control nature of the study necessitates further prospective cohorts to confirm our conclusions. Third, the sample size of this study is relatively small, and further PCR verification is needed in a large sample of people. Fourth, we used target gene prediction and functional enrichment analysis to indirectly obtain the biological processes that may be involved in the pathogenesis, which lacked verification of miRNA's regulation of target genes and cell functions, and further vitro cytology experiments are necessitated.

\section{Conclusions}

This study indicated that miRNAs are differentially expressed in peripheral leukocytes between MMD patients and healthy adults, and among patients with subtypes of MMD. The Wnt signaling pathway is probably involved in the pathogenesis of MMD.

\section{Declarations}

\section{Ethics approval and consent to participate}

The study was performed according to the guidelines from the Helsinki Declaration, and it was approved by the Institutional Review Board (IRB) of the hospital. Written informed consent was obtained from all patients or their legally authorized guardian.

\section{Consent for publication}

Not applicable

\section{Availability of data and material}

The data that support the findings of this study are available from the corresponding author upon reasonable request.

\section{Competing interests}

None

\section{Funding}

This study was supported by the National Science and Technology Major Project (2017ZX09304018), Chinese Academy of Medical Sciences Innovation Fund for Medical Sciences (2019-I2M-5-029), Beijing 


\section{Authors' Contributions}

KK and YS contributed to the conception/design of the study, the acquisition, analysis and interpretation of data, drafting of the manuscript, final approval of the version to be published. QZ, JL, YJ, RJ, NL, JW, $B Y, J L$ and $X L$ were responsible for the acquisition of data, final approval of the version to be published. $X Z$ and DZ were responsible for the conception/design of the study, revision of the work, critical revision of the manuscript, final approval of the version to be published, and agreement to be accountable for all aspects of the work.

\section{Acknowledgments}

We thank all of the patients and health care providers who participated in the present study.

\section{References}

1. Kim JS: Moyamoya Disease: Epidemiology, Clinical Features, and Diagnosis. Journal of stroke 2016, 18(1):2-11.

2. Kuriyama S, Kusaka Y, Fujimura M, Wakai K, Tamakoshi A, Hashimoto S, Tsuji I, Inaba Y, Yoshimoto T: Prevalence and clinicoepidemiological features of moyamoya disease in Japan: findings from a nationwide epidemiological survey. Stroke; a journal of cerebral circulation 2008, 39(1):42-47.

3. Kim T, Lee H, Bang JS, Kwon OK, Hwang G, Oh CW: Epidemiology of Moyamoya Disease in Korea: Based on National Health Insurance Service Data. Journal of Korean Neurosurgical Society 2015, 57(6):390-395.

4. Duan L, Bao XY, Yang WZ, Shi WC, Li DS, Zhang ZS, Zong R, Han C, Zhao F, Feng J: Moyamoya disease in China: its clinical features and outcomes. Stroke; a journal of cerebral circulation 2012, 43(1):56-60.

5. Bao XY, Wang QN, Zhang Y, Zhang Q, Li DS, Yang WZ, Zhang ZS, Zong R, Han C, Duan L: Epidemiology of Moyamoya Disease in China: Single-Center, Population-Based Study. World neurosurgery 2019, 122:e917-e923.

6. Chen PC, Yang SH, Chien KL, Tsai IJ, Kuo MF: Epidemiology of moyamoya disease in Taiwan: a nationwide population-based study. Stroke; a journal of cerebral circulation 2014, 45(5):1258-1263.

7. Bang OY, Fujimura M, Kim SK: The Pathophysiology of Moyamoya Disease: An Update. Journal of stroke 2016, 18(1):12-20.

8. Scott RM, Smith ER: Moyamoya disease and moyamoya syndrome. The New England journal of medicine 2009, 360(12):1226-1237. 
9. Kuroda S, Houkin K: Moyamoya disease: current concepts and future perspectives. The Lancet Neurology 2008, 7(11):1056-1066.

10. Wang Y, Yang L, Wang X, Zeng F, Zhang K, Zhang Q, Liu M, Liu S, Shang M, Li Q et al: Meta-Analysis of Genotype and Phenotype Studies Confirms Predictive Roles of the RNF213 p.R4810K for Moyamoya Disease. European journal of neurology 2020.

11. Wang X, Wang Y, Nie F, Li Q, Zhang K, Liu M, Yang L, Zhang Q, Liu S, Zeng F et al: Association of Genetic Variants With Moyamoya Disease in 13000 Individuals: A Meta-Analysis. Stroke; a journal of cerebral circulation 2020:STROKEAHA120029527.

12. Duan L, Wei L, Tian Y, Zhang Z, Hu P, Wei Q, Liu S, Zhang J, Wang Y, Li D et al: Novel Susceptibility Loci for Moyamoya Disease Revealed by a Genome-Wide Association Study. Stroke; a journal of cerebral circulation 2018, 49(1):11-18.

13. Kang S, Liu X, Zhang D, Wang R, Zhang Y, Zhang Q, Yang W, Zhao JZ: Natural Course of Moyamoya Disease in Patients With Prior Hemorrhagic Stroke. Stroke; a journal of cerebral circulation 2019:STROKEAHA118022771.

14. Kim KM, Kim JE, Cho WS, Kang HS, Son YJ, Han MH, Oh CW: Natural History and Risk Factor of Recurrent Hemorrhage in Hemorrhagic Adult Moyamoya Disease. Neurosurgery 2017, 81(2):289296.

15. Takahashi JC, Funaki T, Houkin K, Inoue T, Ogasawara K, Nakagawara J, Kuroda S, Yamada K, Miyamoto S: Significance of the Hemorrhagic Site for Recurrent Bleeding: Prespecified Analysis in the Japan Adult Moyamoya Trial. Stroke; a journal of cerebral circulation 2016, 47(1):37-43.

16. Morioka M, Hamada J, Kawano T, Todaka T, Yano S, Kai Y, Ushio Y: Angiographic dilatation and branch extension of the anterior choroidal and posterior communicating arteries are predictors of hemorrhage in adult moyamoya patients. Stroke; a journal of cerebral circulation 2003, 34(1):90-95.

17. Liu P, Han C, Li DS, Lv XL, Li YX, Duan L: Hemorrhagic Moyamoya Disease in Children: Clinical, Angiographic features, and Long-Term Surgical Outcome. Stroke; a journal of cerebral circulation 2016, 47(1):240-243.

18. Kuroda S, Kashiwazaki D, Ishikawa T, Nakayama N, Houkin K: Incidence, locations, and longitudinal course of silent microbleeds in moyamoya disease: a prospective T2*-weighted MRI study. Stroke; a journal of cerebral circulation 2013, 44(2):516-518.

19. Mori MA, Ludwig RG, Garcia-Martin R, Brandão BB, Kahn CR: Extracellular miRNAs: From Biomarkers to Mediators of Physiology and Disease. Cell Metab 2019, 30(4):656-673.

20. Dolz S, Górriz D, TembI JI, Sánchez D, Fortea G, Parkhutik V, Lago A: Circulating MicroRNAs as Novel Biomarkers of Stenosis Progression in Asymptomatic Carotid Stenosis. Stroke; a journal of cerebral circulation 2017, 48(1):10-16.

21. Zhao S, Gong Z, Zhang J, Xu X, Liu P, Guan W, Jing L, Peng T, Teng J, Jia Y: Elevated Serum MicroRNA Let-7c in Moyamoya Disease. Journal of stroke and cerebrovascular diseases: the official journal of National Stroke Association 2015, 24(8):1709-1714. 
22. Wang G, Wen Y, Faleti OD, Zhao Q, Liu J, Zhang G, Li M, Qi S, Feng W, Lyu X: A Panel of ExosomeDerived miRNAs of Cerebrospinal Fluid for the Diagnosis of Moyamoya Disease. Front Neurosci 2020, 14:548278.

23. Park YS, Jeon YJ, Lee BE, Kim TG, Choi JU, Kim DS, Kim NK: Association of the miR-146aC > G, miR196a2C > T, and miR-499A > G polymorphisms with moyamoya disease in the Korean population. Neurosci Lett 2012, 521(1):71-75.

24. Dai D, Lu Q, Huang Q, Yang P, Hong B, Xu Y, Zhao W, Liu J, Li Q: Serum miRNA signature in Moyamoya disease. PloS one 2014, 9(8):e102382.

25. Kang HS, Moon YJ, Kim YY, Park WY, Park AK, Wang KC, Kim JE, Phi JH, Lee JY, Kim SK: Smoothmuscle progenitor cells isolated from patients with moyamoya disease: novel experimental cell model. Journal of neurosurgery 2014, 120(2):415-425.

26. Jung KH, Chu K, Lee ST, Park HK, Kim DH, Kim JH, Bahn JJ, Song EC, Kim M, Lee SK et al: Circulating endothelial progenitor cells as a pathogenetic marker of moyamoya disease. Journal of cerebral blood flow and metabolism: official journal of the International Society of Cerebral Blood Flow and Metabolism 2008, 28(11):1795-1803.

27. Willis Rcotpatosootco: Guidelines for diagnosis and treatment of moyamoya disease (spontaneous occlusion of the circle of Willis). Neurologia medico-chirurgica 2012, 52(5):245-266.

28. Zhang Q, Liu Y, Zhang D, Wang R, Zhang Y, Wang S, Yu L, Lu C, Liu F, Zhou J et al: RNF213 as the major susceptibility gene for Chinese patients with moyamoya disease and its clinical relevance. $J$ Neurosurg 2016:1-8.

29. Mahajan SG, Fender AC, Meyer-Kirchrath J, Kurt M, Barth M, Sagban TA, Fischer JW, Schrör K, Hohlfeld T, Rauch BH: A novel function of FoxO transcription factors in thrombin-stimulated vascular smooth muscle cell proliferation. Thrombosis and haemostasis 2012, 108(1):148-158.

30. Tsaousi A, Williams H, Lyon CA, Taylor V, Swain A, Johnson JL, George SJ: Wnt4/beta-catenin signaling induces VSMC proliferation and is associated with intimal thickening. Circulation research 2011, 108(4):427-436.

31. Foulquier S, Daskalopoulos EP, Lluri G, Hermans KCM, Deb A, Blankesteijn WM: WNT Signaling in Cardiac and Vascular Disease. Pharmacol Rev 2018, 70(1):68-141.

32. Menet R, Lecordier S, ElAli A: Wnt Pathway: An Emerging Player in Vascular and Traumatic Mediated Brain Injuries. Front Physiol 2020, 11:565667.

33. Shao Y, Chen J, Freeman W, Dong LJ, Zhang ZH, Xu M, Qiu F, Du Y, Liu J, Li XR et al: Canonical Wnt Signaling Promotes Neovascularization Through Determination of Endothelial Progenitor Cell Fate via Metabolic Profile Regulation. Stem Cells 2019, 37(10):1331-1343.

\section{Figures}




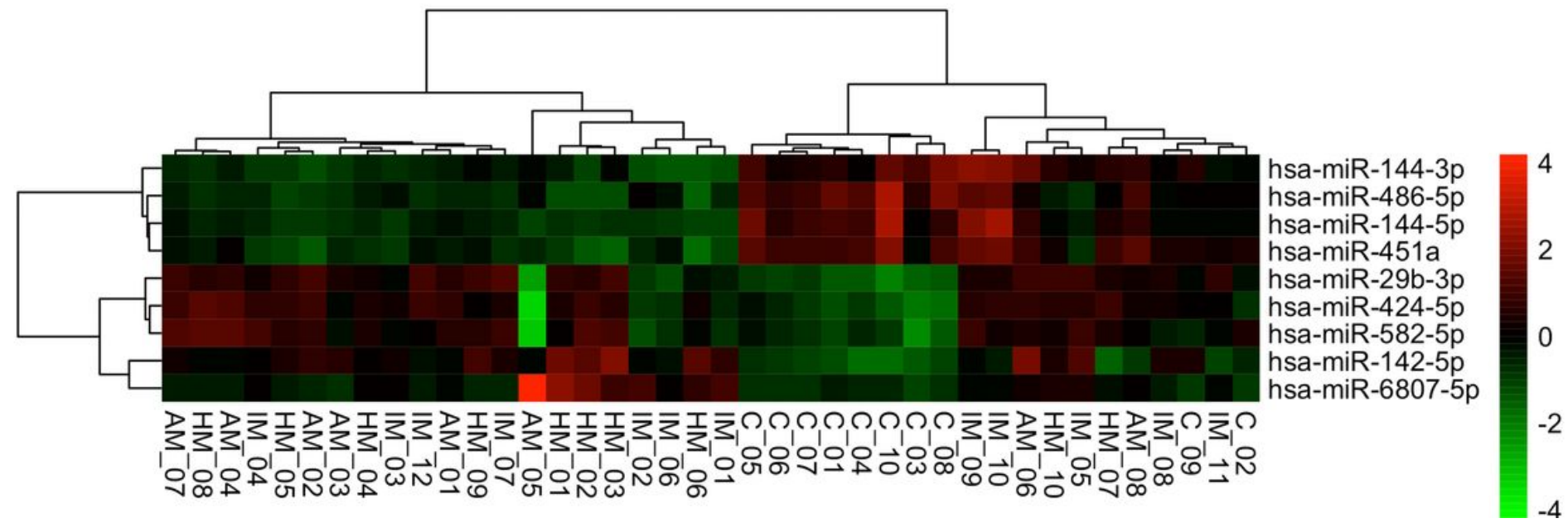

Figure 1

Heat map of differentially expressed miRNAs between MMD patients and healthy controls 

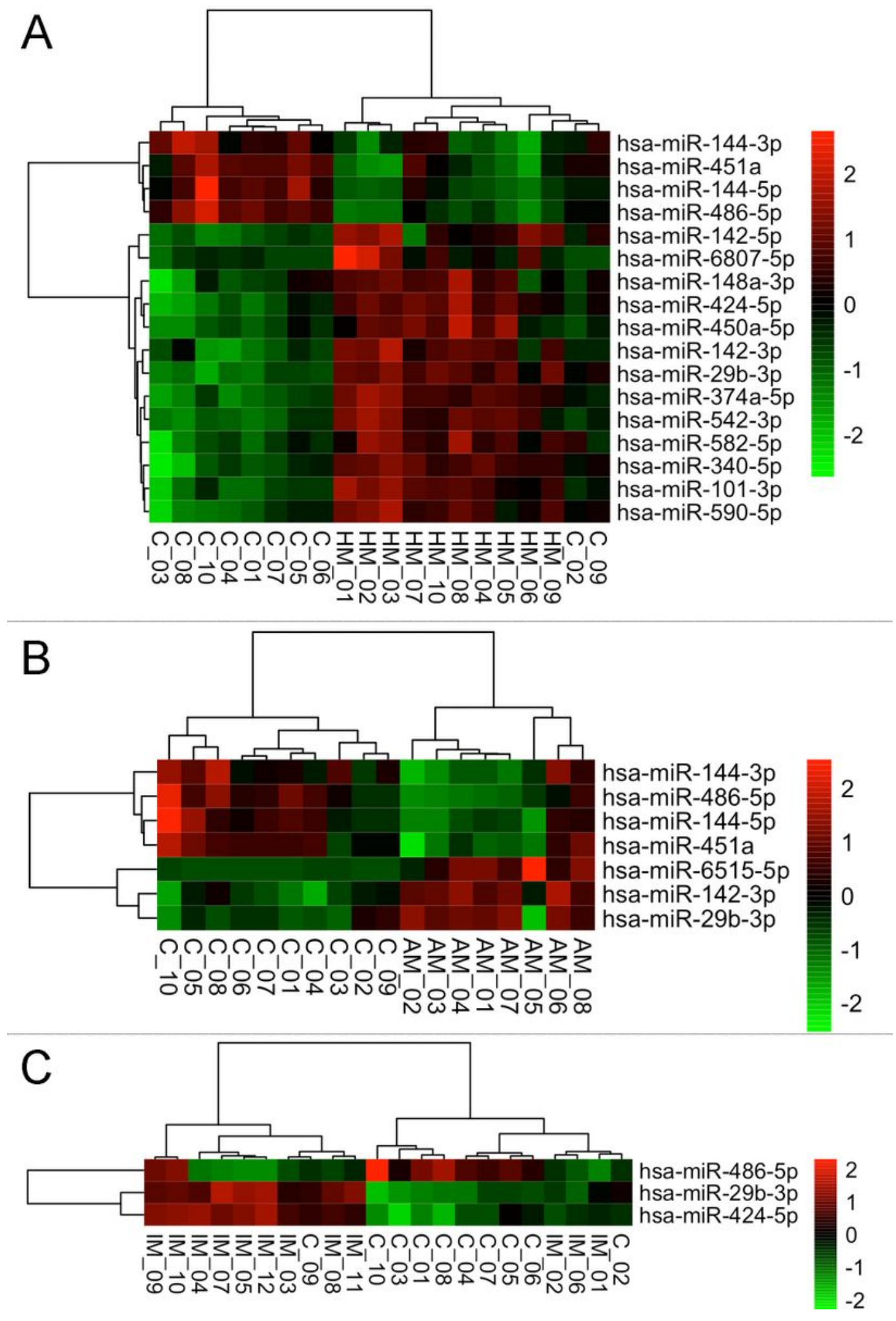

Figure 2

Heat map of differentially expressed miRNAs between hemorrhagic MMD patients (A), ischemic (B), asymptomatic MMD patients (C) and healthy controls 


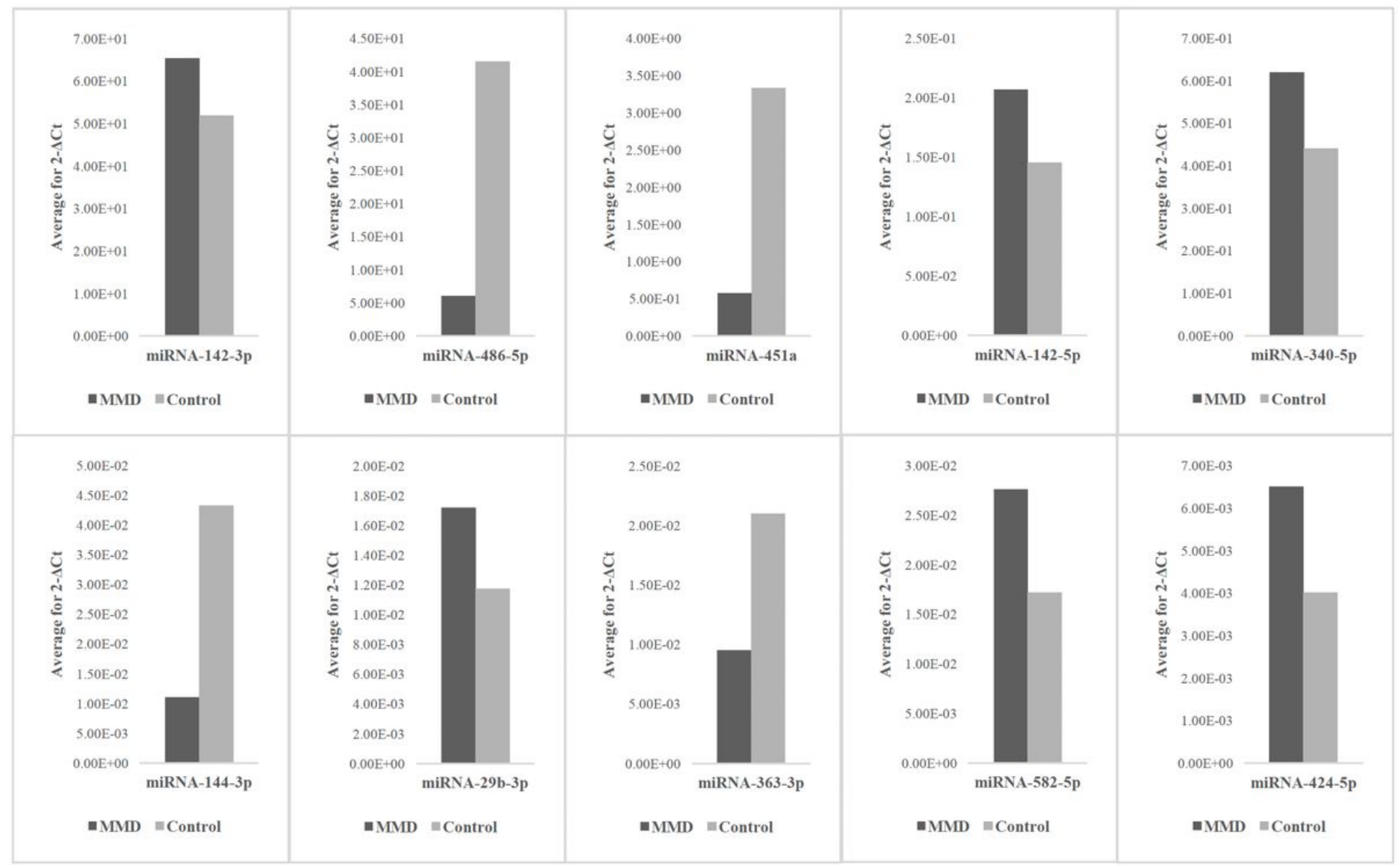

\section{Figure 3}

Validation of microarray results using quantitative real-time PCR

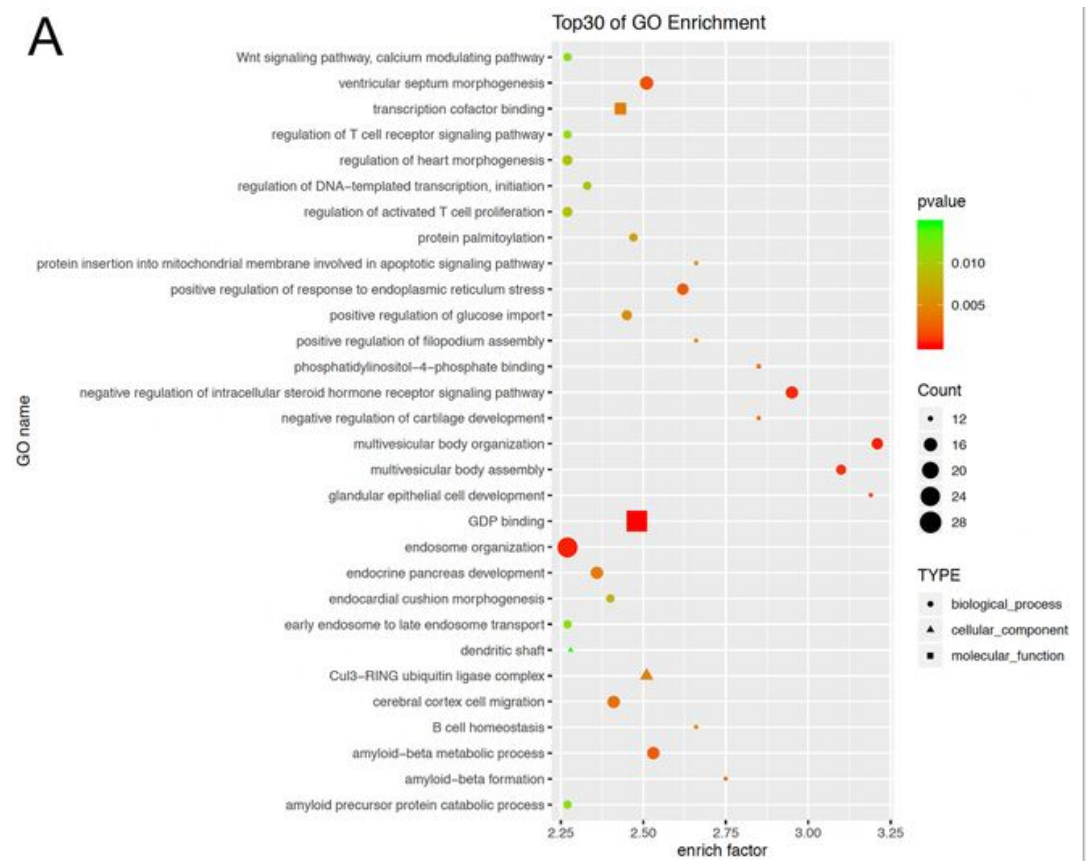

$B$

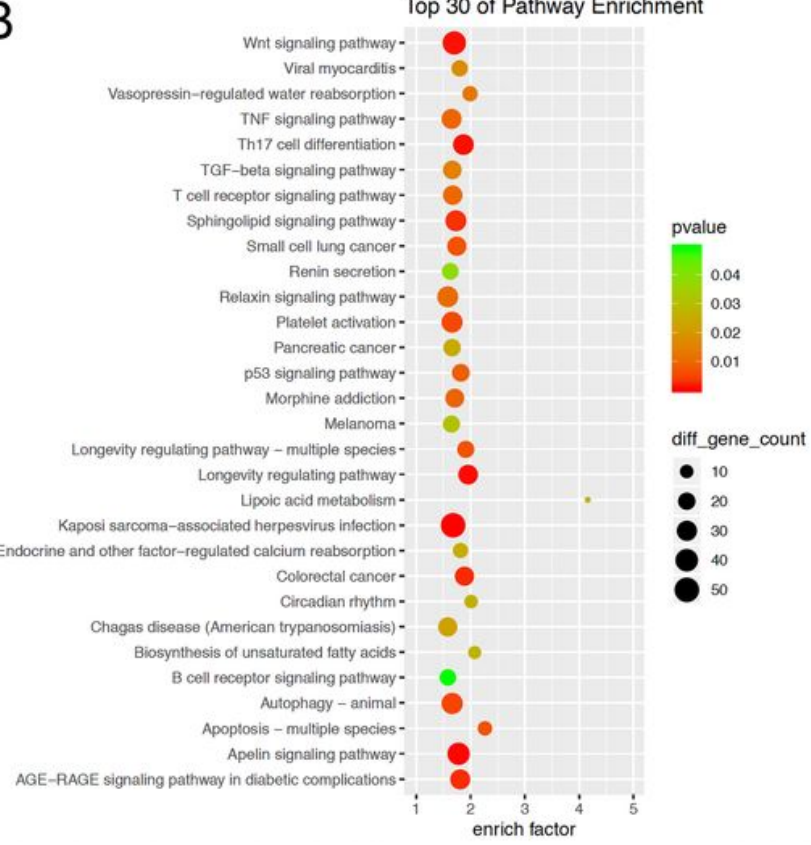

Figure 4 


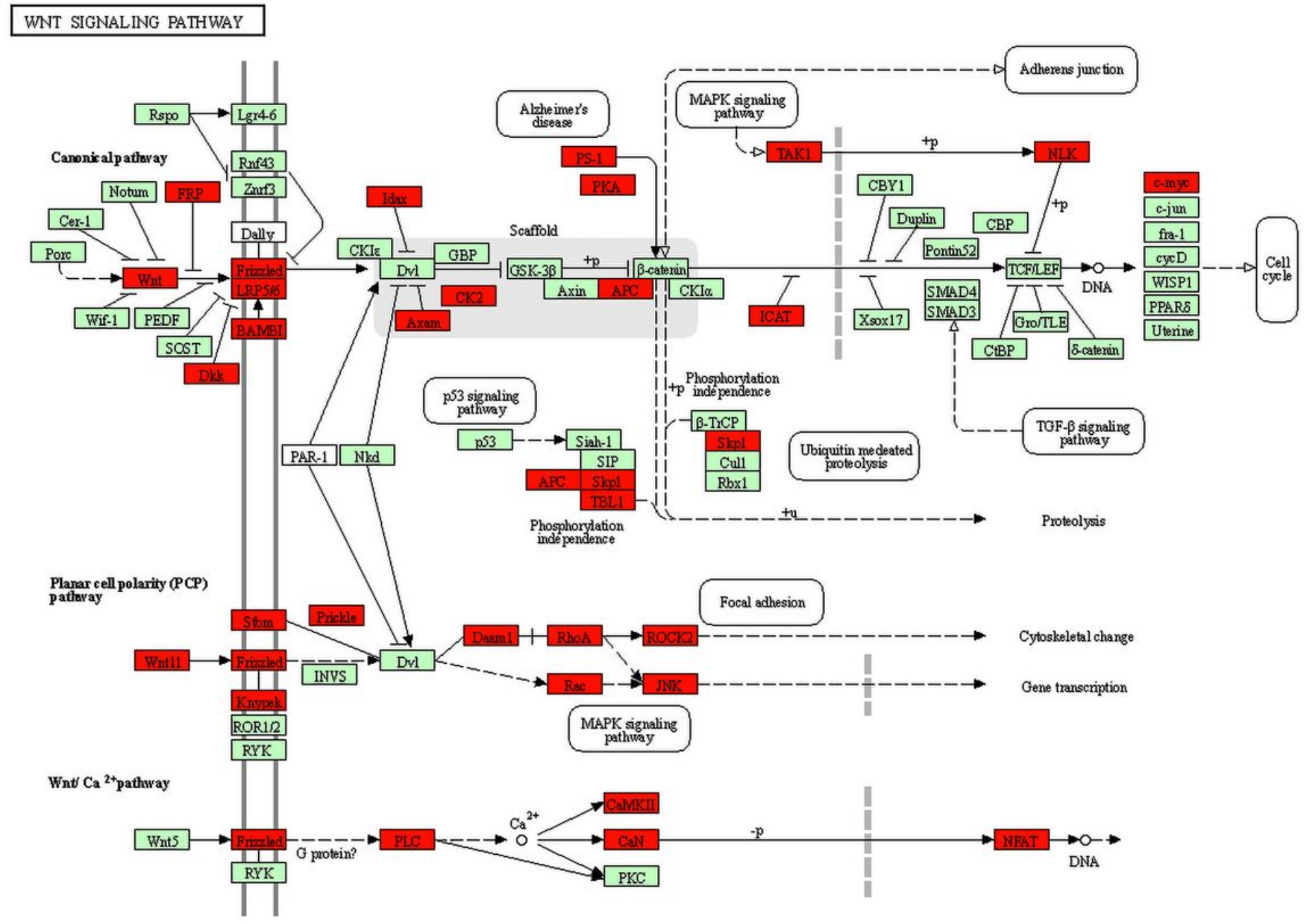

\section{Figure 5}

Target genes (highlighted in red) in the KEGG Wnt pathway map generated using DAVID software 